\title{
Training by ART-2 and Classification of Ballistic Missiles Using Hidden Markov Model
}

\author{
Upendra Kumar Singh ${ }^{1}$ and Vineet Padmanabhan ${ }^{2}$ \\ 1 PGAD, Defence Research \& Development Organisation, Hyderabad, India \\ singh_ukin@yahoo.co.in \\ 2 School of Computer \& Information Sciences, University of Hyderabad, India \\ vineetcs@uohyd.ernet.in
}

\begin{abstract}
This paper addresses the classification of different ranges of Ballistic Missiles (BM) for air defense applications using Adaptive Resonance Theory (ART-2) and Hidden Markov Model (HMM). ART-2 finds the initial clusters using unsupervised learning to be fed to HMM for classification using recursive method. The classification is based on derived parameters of specific energy, acceleration, altitude and velocity which in turn are acquired from measured data by radars. To meet the conflicting requirements of classifying short as well as long-range BM trajectories, we are proposing a formulation for partitioning the trajectory by using a moving window concept. Experimental results show that the HMM model is able to classify above $95 \%$ within time of the order of milliseconds once initial data is trained using ART2.
\end{abstract}

\section{Introduction}

In this work, we deal with the classification of ballistic missiles in real-time for air-defense application. The classification of ballistic missile trajectory is a challenging problem due to its time-varying dynamics and short response time available for interception. The general policy of Ballistic missile defense is to neutralize incoming threats at higher height and at longer range so that debris falls away from intended impact zone [8]. This may not be realized due to late detection and short response time of launch. In a typical ballistic missile defense (BMD) scenario the radar first detects the target missile and information is communicated to the launcher. The launcher in-turn classifies the target to decide about the time of launch and height of interception. The response time is dependent on many factors including range of radars for acquiring target of the given radar cross section (RCS), target velocity, preparation time of interceptor, velocity of interceptor, time of flight of interceptor, planned height, range of kill and classification time. Classification of variable-length trajectory and time varying dynamic attributes, which are important ingredients for ballistic missile classification, cannot be done using conventional techniques like neural networks, Bayesian or Kernel methods [2]. Classification of motion trajectories using Hidden Markov Models (HMMs) is presented in [9] and Gaussian mixturebased HMMs for trajectory modeling and classification are described by Bashir,

P. Maji et al. (Eds.): PReMI 2013, LNCS 8251, pp. 108-114, 2013.

(C) Springer-Verlag Berlin Heidelberg 2013 
et.al [1]. In this paper a novel procedure is formulated to group initial data using unsupervised ART-2 algorithm and then classify ballistic missiles using HMM.

\section{Problem Formulation}

The radar measures only the position of the target and this information is noisy [11. The kalman filter estimates position, velocity and acceleration by processing the noisy measured position data. The filtered kinematic parameters of missiles are passed to linear quantizer to convert the data into discrete forms before it passes through HMM. For the present work, we are using 4 kinematic parameters, which are computed based on the kalman filter estimates. These 4 parameters are (a) Specific energy, (b) Acceleration, (c) Altitude, and (d) Velocity. Also the number of classes considered are three, namely M400 Class of Ballistic Missile (400 Km range), M1000 Class of Ballistic Missile (1000 Km range) and M2000 Class of Ballistic Missile (2000 Km range). HMM parameters are initialised, and Baum-Welch (BW) algorithm is applied to get a new set of parameters of HMM that has maximum likelihood of occurrence, given a set of observed feature vectors. Running many iterations of BW yields a sequence that approaches a local maximum of the likelihood. The Viterbi algorithm finds the most probable way through the model $\Lambda$ and evaluate the model by maximising probability of correspondence with a trajectory class. Hidden Markov Model (HMM) is trained for a set of points of the trajectory and evaluated to assess the performance of the model using trajectories of ballistic missiles. The training time taken for computation is less than $100 \mathrm{~ms}$ and it is highly suited for applications where scene of interest is changing continuously and next state vector is dependent on only a limited last states within a set window. However, initial set of data needed for HMM has to be pre-specified or known using another algorithm. ART-2, a competitive learning algorithm, is used here for self-organising input pattern based on user defined parameters of the network.

Since ballistic missiles can be programmed to fly in nominal, lofted and depressed mode the parameters used for classification varies for same as well as different classes of target missiles. To meet the conflicting requirements of classifying short as well as long range trajectories, a formulation is proposed for partitioning the trajectory by using moving window concept. This concept allows us to use parameters in localized frame which helps in reducing the problem to fit into the same model. This moving window concept of testing and training brings out the knowledge embedded in the system and deals with variable length inputs. The length of the moving window is selected such that it fully captures the dynamics of the target which are intended to be classified.

\section{Adaptive Resonance Theory (ART2)}

An adaptive resonance theory (ART) 5] involves three groups of neurons: input processing units (F1 layer), cluster units (F2 layer), and reset units. The F1 layer consists of six types of units (W, X, U, V, P and Q). There are ' $\mathrm{n}$ ' (dimension 


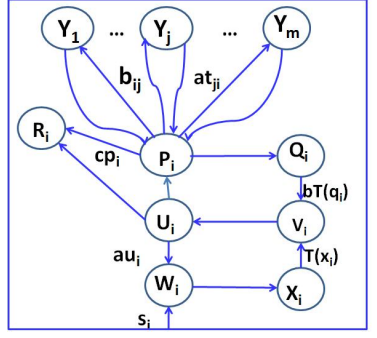

Fig. 1. ART2 Architecture

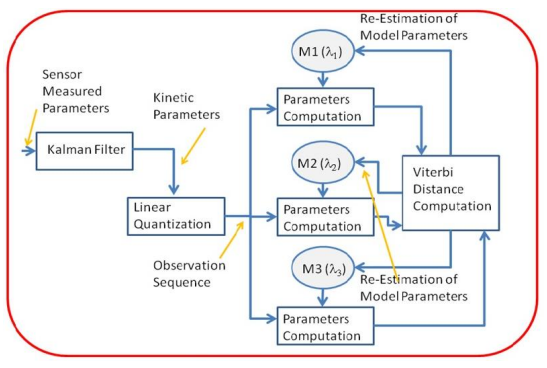

Fig. 2. HMM Recogniser

of input pattern) units of each of these types whereas Figure 1 shows only one unit. $S_{i}$ forms the input vector. Layer F2 is shown as units $Y_{1}, \ldots, Y_{m}$. There are two sets of connections between the layers F1 and F2. The bottom-up weights connecting F1 to F2 are denoted by $b_{i j}$ and the top-down weights connecting $\mathrm{F} 2$ to $\mathrm{F} 1$ are designated $t_{j i}$. The cluster unit with the largest net input becomes the candidate to learn the input vector. The criterion for an adequate match between an input pattern and a chosen cluster (feature) is determined by a vigilance parameter, which ranges between 0 and 1 . The units $V_{i}$ and $P_{i}$ in the F1 layer send signals to the corresponding reset unit $R_{i}$. The reset mechanism can check for a reset each time it receives a signal from $P_{i}$. The steps involved in ART-2 are (1) As each input is presented to the network, it is compared with the prototype vector that matches most closely. (2) If the match between the prototype and the input vector is NOT adequate, a new prototype is selected. In this way, previous learned memories are not eroded by new learning. The ART2 network is used for classifying initial set of samples to be utilised further for HMM beyond 20 samples. A typical ART2 architecture [3] is re-produced in Figure 1] The parameters chosen for the network for training initial set of values have been fixed after experiment and tabulated in Table 1.

\section{Hidden Markov Model (HMM)}

HMM is a statistical method which is rich in mathematical structure and forms basis for use in a wide range of applications [974. A trajectory is a continuous quantity which can be described as the position of the object in time. HMMs are finite state stochastic machines that robustly model temporal variations in time series data which satisfies the Markovian property [6]. The basic theoretical strength of the HMM is that it combines modeling of stationary stochastic processes and the temporal relationship among the processes together in a well-defined probability space [10]. Considering the trajectory of missile of a particular class to be described at any time as being in one of a set of $\mathrm{N}$ distinct states $S_{1}, S_{2}, \ldots, S_{N}$. At regularly spaced discrete times, the system undergoes a change of state according to a set of probabilities associated with the state. Denoting the time instants associated with the state changes as $t=1,2, \ldots$, the actual state at time $t$ is denoted as $q_{t}$. Main characteristics of HMM are 1) Given 
Table 1. Choice of parameters of ART2

\begin{tabular}{|l|l|l|l|}
\hline $\begin{array}{l}\text { Name of } \\
\text { Parameters }\end{array}$ & Values & Name of parameters & Values \\
\hline $\begin{array}{l}\text { No. of Inputs } \\
(\text { F1 Layer })\end{array}$ & 4 & Vigilance Parameter $(\rho)$ & 0.999985 \\
\hline $\begin{array}{l}\text { No. of Inputs } \\
(\text { F2 Layer })\end{array}$ & 4 & Fixed weights in the F1 layer $(\mathrm{a}, \mathrm{b})$ & 10,10 \\
\hline No. of Epochs & 1 & Fixed weight used for reset $(\mathrm{c})$ & 0.1 \\
\hline No. of Iterations & 200 & Activation of winning F2 unit $(\mathrm{d})$ & 0.9 \\
\hline Learning rate $(\alpha)$ & 0.1 & Noise suppression parameter $(\theta)$ & $\frac{1}{\operatorname{sqrt}(n)}$ \\
\hline $\begin{array}{l}\text { Initial top-down } \\
\text { weights } t_{j i}\{O\}\end{array}$ & 0 & Initial bottom-up weights $b_{i j}\{O\}$ & $\frac{1}{((1-d) \operatorname{sqrt}(n))}$ \\
\hline
\end{tabular}

the $(t-1)^{t h}$ state variable, the $t^{t h}$ state variable is independent of previous variables and 2) the $t^{t h}$ observation depends only on the $t^{t h}$ state.

The trajectory classification problem is formulated as to identify the class $C_{i}(i=$ $1 \ldots L)$ to which the trajectory state sequence belongs. Each trajectory class $C_{i}$ is represented by a Model, $\lambda_{i}$, where $\lambda_{i}=\{\pi, A, B\}$ in which $\pi$ is initial state probabilities, $A$ is state transition probabilities of dimension $(\mathrm{N} \times \mathrm{N})$ and $\mathrm{B}$ is observation symbol probability distribution of dimension $(\mathrm{N} \times \mathrm{M})$. Number of states for HMM for Missile classification are considered as $\mathrm{N}=25$; Number of Observation Symbols, M for Specific Energy is 200, Acceleration is 40, Height is 60 and Velocity is 80 . Initial state probability, $\pi$ and state transition probabilities are set to be equal to $1 / \mathrm{N}$. Observation symbol probability, B is based on Gaussian (with mean and variance) representation for the distributions of observations within each state. HMM recognizer as given in Figure 2 shows three HMM models $\left(\lambda_{1}, \lambda_{2}, \lambda_{3}\right)$ corresponding to three different types of trajectories belonging to M400, M1000 and M2000 class of missiles. Radar measured data is passed through the Kalman filter as indicated by first block. Observation sequence is linearly quantized and passed through Baum-Welch 2,10] computation to arrive at updated parameters of HMM Model. Viterbi [2 10] distance is used to classify how a new sample is related with trained HMM Model. The steps involved in unified training and testing can be summarized as follows: 1) Define all normal trajectory classes in the scene 2) Define Initial models corresponding to each class 3) The trajectory classes in Captured sequence are marked with 20 samples of data and models are adapted according to them using Baum-Welch Algorithm 4) The output is a new classification model 5) New set of data is evaluated against each HMM model using Viterbi Distance 6) Least Viterbi distance is taken as criteria to assign new data to the corresponding Class 7) New data is added at the end of running window of that class \& first data is deleted 8) Training is repeated to adapt the Models 9) Step (5) to (8) is repeated for all new set of data. Termination criteria of BW iteration occurs if maximum 
changes for consecutive iteration for all three $\{\pi, A, B\}$ parameters are less than $10 e-8$. Re-estimation formulas for $\{\pi, A, B\}$ are given in [10].

\section{Results and Analysis}

Input data is either taken from radar for live target or generated from mathematical six degree-of-freedom (6-DOF). Sub-modules of 6 DOF are propulsion, gravity, aero-dynamics, atmospheric, missile configuration. External disturbance due to wind and misalignment is also considered in the model. Runga-Kutta order 4 is used for solving the 6 equations of 6 DOF i.e. 3 for translational and 3 for rotational motions of the body. If data are taken from simulation, noise is added as per the radar specification to make it near realistic scenario, where azimuth and elevation channels are corrupted with 2 milli-radians and range by $20 \mathrm{~m}$. The measurement data is passed through kalman filter to estimate the target state i.e. position, velocities and acceleration. Based on this estimated data the kinematic parameters, namely specific energy, acceleration, altitude and velocity as mentioned in Section-2 are derived and fed with uniform track rate update of 100ms. Figure 3(a), Figure 3(b) and Figure 4(a) shows Viterbi distance of
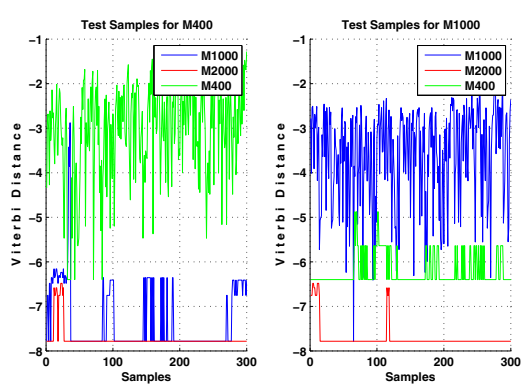

Fig. 3. HMM Results a) M400 b) M1000 for 300 samples for each
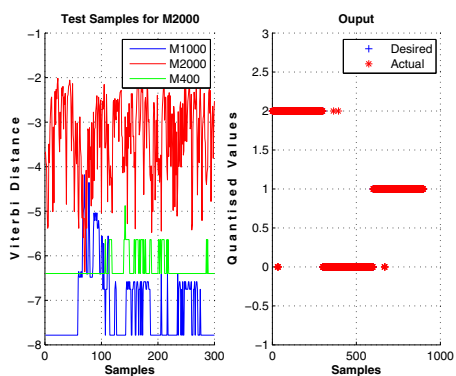

Fig. 4. HMM Results a) M2000 for 300 samples b) Class Category

three classes of missiles as new samples are arriving and being fed to the model for a total of 900 samples for three types of missiles, each with 300 samples. These figures clearly show that trajectory under test are segregated from other two classes for most of the duration. However, at some points, few samples of M1000 trajectory are misclassified as M400 class. Figure 4(b) shows how well desired and computed outputs are matching. Desired symbols for M400, M1000 and M2000 classes are $(2,0,1)$ respectively where a symbol count is incremented during testing if computed values finds matches with correct class. This figure shows that for most of the duration, desired and computed values are overlapping except for very small duration. Results of HMM as recorded in Table-2 demonstrate that pass percentage achieved is more than $95.5 \%$ over 900 samples of test data. However, misclassification is ranging from zero to $4.5 \%$. Diagonal values of Table 2 show correct classification of three types of missiles. Off-diagonal values 
Table 2. Performance of HMM Model in Confusion Matrix Form

\begin{tabular}{|c|c|c|c|c|}
\hline \multicolumn{2}{|c|}{} & \multicolumn{3}{|c|}{ Actual Class } \\
\cline { 3 - 5 } \multicolumn{2}{|c|}{} & Class 1 & Class 2 & Class 3 \\
\hline \multirow{3}{*}{$\begin{array}{c}\text { Predicted } \\
\text { class }\end{array}$} & Class 1 (M1000) & $298 / 300$ & 4 & 5 \\
\cline { 2 - 5 } & Class 2 (M2000) & 0 & $296 / 300$ & 0 \\
\cline { 2 - 5 } & Class 3 (M400) & 2 & 0 & $295 / 300$ \\
\hline
\end{tabular}

shows misclassification. HMM takes $1.234 \mathrm{sec}$ for training of 20 samples of initial values in addition to overheads for output storage. HMM takes $44.319 \mathrm{sec}$ of time for unified testing and training of 900 samples which brings $49.353 \mathrm{~ms}$ for one sample. The trajectory is validated on a model with different number of states in HMM. HMM with 20 states, 25 numbers of states and above are giving results above $95 \%$ whereas performance of the model remains unsatisfactory with 5,10 or 15 states. Time taken by the model for testing new sample of data for 5,10,15,20 and 25 states are 4.816 milli-seconds (ms), $8.02 \mathrm{~ms}, 18.17 \mathrm{~ms}, 29.06$ $\mathrm{ms}$ and $49.24 \mathrm{~ms}$ respectively.

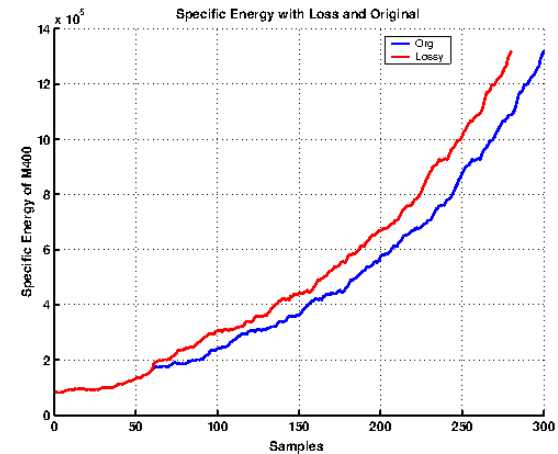

Fig. 5. Trajectory with 2 seconds loss

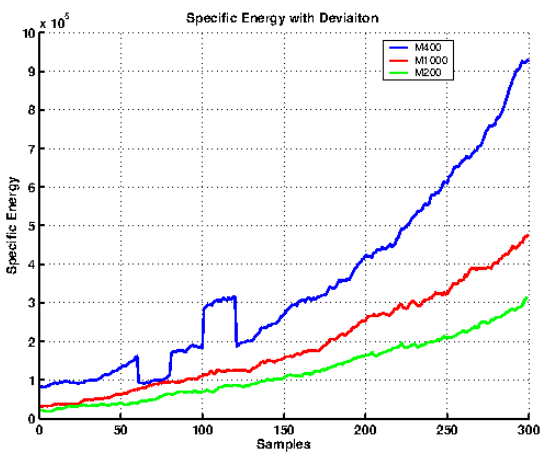

Fig. 6. Trajectory with deviation

The formulation is also tested with two important and practical conditions of losses of data (figure 5) in the track and manoeuvrings targets (figure 6), later being a recent improvement in the ballistic missile development. Figure 5 shows specific energy (SE) plot of M400 class of missile with original and with loss of 20 samples of data equivalent to 2 seconds. The model continues to classify the input data correctly visible as diagonal elements of matrix of Table 3 . Numerator indicates model output and denominator denotes total samples passed for validation. Performance of the model is correct classification of $99.31 \%$ and incorrect classification for $0.68 \%$ for all 3 classes. Results for manoeuvring target are demonstrated as in Table 4. Correct cumulative classification of $97.24 \%$ is achieved, whereas incorrect classification amounts to $2.76 \%$. Class 3 for M400 in case of deviated trajectory is passing with $95.29 \%$ of correctness. 
Table 3. Results with 20 samples Loss

\begin{tabular}{|c|c|c|c|c|}
\hline \multicolumn{2}{|c|}{} & \multicolumn{3}{|c|}{ Actual Class } \\
\cline { 3 - 5 } \multicolumn{2}{|c|}{} & Class 1 & Class 2 & Class 3 \\
\hline \multirow{2}{*}{$\begin{array}{l}\text { Pred- } \\
\text { icted } \\
\text { class }\end{array}$} & M1000 & $296 / 300$ & 0 & 0 \\
\cline { 2 - 5 } & M2000 & 4 & $298 / 300$ & 0 \\
\hline
\end{tabular}

Table 4. Trajectory deviation results

\begin{tabular}{|l|c|c|c|c|}
\hline \multicolumn{2}{|c|}{} & \multicolumn{3}{|c|}{ Actual Class } \\
\cline { 2 - 5 } \multicolumn{2}{|c|}{} & Class 1 & Class 2 & Class 3 \\
\hline \multirow{2}{*}{\begin{tabular}{l} 
Pred- \\
\multirow{2}{*}{$\begin{array}{l}\text { icted } \\
\text { class }\end{array}$}
\end{tabular}} & M1000 & $296 / 300$ & 0 & 0 \\
\cline { 2 - 5 } & M400 & 0 & $291 / 300$ & 16 \\
\hline
\end{tabular}

\section{Conclusions}

In this work, we have presented a framework for classification of Ballistic Missiles trajectory using ART-2 and HMM in real-time. Competitive learning of ART2 categorise the incoming samples into groups to be utilised by HMM for dynamic testing a new sample for its class and clubbing with classified group for training in the subsequent cycles. 6 DOF model generates trajectories of different ranges of ballistic missiles, which is utilized for testing and evaluation of ART2 and HMM. Making window of fix length allows HMM to integrate testing and training while taking care of variety of trajectories to be addressed by the same model. Further, we have established that the HMM is able to classify the new sample in less than 50 milliseconds which makes it to use for real-time applications with above $95 \%$ success rate on Pentium-4 processor.

\section{References}

1. Bashir, F.I., Khokhar, A.A., Schonfeld, D.: Object trajectory-based activity classification and recognition using hidden markov models. IEEE Transactions on Image Processing 16(7), 1912-1919 (2007)

2. Bishop, C.M.: Pattern reccognition and Machine Learning. Springer (2006)

3. Fausett, L.: Fundamentals of Neural Networks - Architectures, Algorithms and Applications. Prentice Hall (2008)

4. Fraser, A.M.: Hidden Markov Models and Dynamical Systems. Society for Industrial and Applied Mathematics (2008)

5. Carpenter, G.A., Grossberg, S.: Art 2: self-organization of stable category recognition codes for analog input patterns. Journal of Optics 26(23), 4919-4930 (1987)

6. Garcia, J., Poncha, O.P., Molina, J.M., de Miguel, G.: Trajectory classification based on machine-learning techniques over tracking data. In: 9th International Conference on Information Fusion, pp. 1-8 (2006)

7. Juang, B.H., Rabiner, L.R.: Hidden markov models for speech recognition. Technometrics 33(3), 251-272 (1991)

8. Mantle, P.J.: The Missile Defense Equation-Factors for decision making. American Institute of Aeronautics and Astronautics (2003)

9. Mlich, I.J., Chmelar, P.: Trajectory classification based on hidden markov models. In: 18th International Conference on Computer Graphics and Vision, pp. 101-105 (2008)

10. Rabiner, L.R.: A tutorial on hidden markov models and selected applications in speech recognition. Proceedings of IEEE 77(2), 257-286 (1989)

11. Tait, P.: Introduction to Radar Target Recognition. The Institution of Engineering and Technology (2005) 